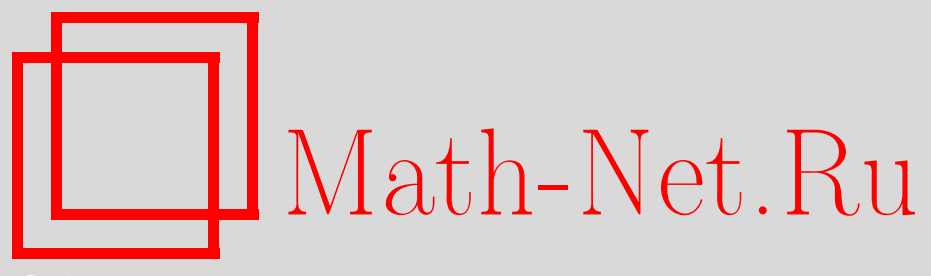

Д. А. Славнов, О возможности согласования квантовой механики с классической теорией вероятностей, ТMФ, 2006, том 149, номер 3, 457-472

DOI: https://doi.org/10.4213/tmf5537

Использование Общероссийского математического портала Math-Net.Ru подразумевает, что вы прочитали и согласны с пользовательским соглашением http://www . mathnet.ru/rus/agreement

Параметры загрузки:

IP : 3.85 .5 .30

26 апреля 2023 г., 16:27:45

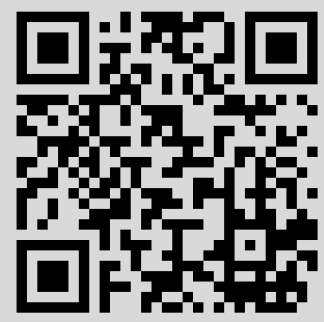




\title{
О ВОЗМОЖНОСТИ СОГЛАСОВАНИЯ КВАНТОВОЙ МЕХАНИКИ С КЛАССИЧЕСКОЙ ТЕОРИЕЙ ВЕРОЯТНОСТЕЙ
}

\begin{abstract}
Описана схема построения квантовой механики, в которой квантовая система рассматривается как совокупность открытых классических подсистем. Это позволяет использовать в квантовой механике формальную классическую логику и классическую теорию вероятностей. С другой стороны, в рамках такого подхода удается полностью воссоздать стандартный математический аппарат квантовой механики, указав границы его применения. Специально рассматривается проблема редукции квантового состояния.
\end{abstract}

Ключевые слова: квантовое измерение, алгебра наблюдаемых, теория вероятностей, редукция квантового состояния.

\section{1. ВВЕДЕНИЕ}

Пионеры квантовой механики при создании новой теории основывались на очень небольшом количестве экспериментальных фактов. В результате квантовая механика оказалась скорее некоторой математической схемой (хотя и очень удачной), чем физической моделью. Не случайно предложенный Гейзенбергом вариант квантовой теории получил название "матричная механика". Таким образом, ключевым понятием физической теории оказался чисто математический объект - матрица.

Волновая механика Шредингера задумывалась как физическая модель. Основному понятию этой модели, волновой функции, сам Шредингер настойчиво пытался придать физический смысл. Но из этого ничего не получилось. Пришлось смириться с тем, что волновая функция - это амплитуда вероятности. В лучшем случае, это полезный математический объект, который непосредственного физического смысла не имеет.

Логическое завершение эта тенденция развития квантовой механики получила в знаменитой книге фон Неймана [1]. Фактически роль квантовой механики свелась к физической интерпретации теории гильбертова пространства.

* Московский государственный университет, Москва, Россия. E-mail: slavnov@goa.bog.msu.ru 
С одной стороны, это позволило придать квантовой механике четкую математическую структуру и развить мощный математический аппарат. С помощью этого аппарата оказалось возможным математически описать огромное число физических явлений. С другой стороны, физики практически отказались от попыток понять физическую сущность квантовых явлений.

Квантовая механика превратилась в некоторый “черный ящик". В этот ящик следует закладывать исходные данные. Он их перерабатывает по физически непонятным законам и выдает ответ, который можно сравнивать с результатами эксперимента. В подавляющем числе случаев получается очень хорошее согласие.

С прикладной точки зрения, как правило, этого достаточно. Но, по большому счету, это не задача естественной науки, а скорее задача инструкции для пользователя. Недаром из уст крупнейших специалистов в области квантовой физики можно порой услышать такие высказывания: “Квантовую механику можно выучить, можно научиться получать из нее полезные результаты, но понять ее нельзя".

Приоритет математики в построении квантовой механики потенциально опасен еще и по следующей причине. Желание построить математически совершенную схему заставляет исследователя делать предположения и накладывать условия, которые выглядят очень естественными с точки зрения математики и при этом оказываются достаточными для правильного описания рассматриваемого физического явления. В этом случае такие предположения, как правило, воспринимаются в качестве физических. Между тем необходимость для физики этих математических условий обычно остается вне поля зрения.

Математические предположения могут приводить и к незапланированным физическим следствиям, которые не имеют непосредственного экспериментального подтверждения. Например, наличие траектории у квантовой частицы противоречит математическому аппарату стандартной квантовой механики. Вместе с тем всегда, когда удается проследить перемещение квантовой частицы в пространстве, такая траектория обнаруживается. Конечно, приводятся более или менее убедительные доводы, почему мы не можем обнаружить отсутствие траектории. Но прямого экспериментального подтверждения этому факту нет.

Приведенные соображения ни в коем случае не надо воспринимать как призыв к ограничению использования математики в физике и, в частности, в квантовой механике. Однако надо всячески стараться, чтобы при построении физической теории математика следовала за физикой, а не наоборот. На практике это означает, что надо отталкиваться от физического явления. На втором этапе, когда дается математическое описание этого явления, надо заботиться не только о том, чтобы математические предположения были достаточными для такого описания, но и стремиться ограничиться только необходимыми с физической точки зрения предположениями. Только при выполнении условия необходимости можно быть более или менее уверенным, что физические следствия из этих математических предположений реализуются в природе. 
Конечно предложенная схема является идеальной и в чистом виде никогда не реализуется, но к ней надо стремиться. Далее при формулировке квантовой механики мы по мере сил будем пытаться следовать этой программе.

\section{2. НАБЛЮДАЕМЫЕ, ИЗМЕРЕНИЯ, СОСТОЯНИЯ}

Базовым понятием как классической, так и квантовой физики является наблюдаемая. Наблюдаемая - это такой атрибут физической системы, для которого с помощью определенной измерительной процедуры можно получить некоторое численное значение. Далее будем считать все наблюдаемые безразмерными. Это означает, что у нас зафиксирована некоторая система единиц.

При каждом измерении исследуемая физическая система подвергается воздействию со стороны измерительного прибора. Поэтому все измерения мы можем разделить на два типа: воспроизводимые и невоспроизводимые. Характеристической особенностью воспроизводимых измерений является то, что при повторном измерении той же наблюдаемой мы получаем первоначальное значение.

Особо остро стоит вопрос о воспроизводимости при измерении нескольких наблюдаемых для одной физической системы. Пусть мы измеряем наблюдаемую $\hat{A}$, затем наблюдаемую $\widehat{B}$, потом опять наблюдаемую $\hat{A}$ и, наконец, повторно наблюдаемую $\widehat{B}$. Если в результате таких повторных измерений для каждой из наблюдаемых мы будем получать повторяющиеся значения, то назовем измерения наблюдаемых $\hat{A}$ и $\widehat{B}$ совместимыми.

Опыт показывает, что коренное различие классических и квантовых физических систем заключается в следующем. Для классических систем всегда можно организовать эксперимент так, что измерения двух любых наблюдаемых будут совместимыми. У квантовой системы всегда найдутся такие наблюдаемые, для которых ни при каких обстоятельствах нельзя провести совместимые измерения. Такие наблюдаемые называются несовместимыми. Соответственно совместимыми, или одновременно измеримыми, называются наблюдаемые, для которых совместимые измерения провести можно. Приборы, с помощью которых можно провести совместимые измерения, назовем совместимыми. Для несовместимых наблюдаемых такие приборы не существуют.

Обозначим множество всех наблюдаемых для рассматриваемой физической системы через $\mathfrak{A}_{+}$, а его максимальное подмножество совместимых наблюдаемых через $\mathfrak{Q}_{\xi}$. Ясно, что для классической системы такое подмножество одно - это само множество $\mathfrak{A}_{+}$. Для квантовой системы должно быть не менее двух таких подмножеств. Можно убедиться, что их бесконечно много [2]. Индекс $\xi$, который пробегает множество $\Xi$, отличает одно такое подмножество от другого. Одна и та же наблюдаемая может одновременно принадлежать различным подмножествам $\mathfrak{Q}_{\xi}$.

Легко убедиться, что каждое из подмножеств $\mathfrak{Q}_{\xi}$ можно оснастить структурой действительной коммутативной ассоциативной алгебры. В самом деле, опыт показывает, что для любых совместимых наблюдаемых $\hat{A}$ и $\widehat{B}$ существует третья наблюдаемая $\widehat{D}$, которая, во-первых, совместима с наблюдаемыми $\hat{A}$ и $\widehat{B}$, во-вторых, при каждом одновременном (совместимом) измерении этих трех наблюдаемых для 
результатов измерения мы будем получать соотношение

$$
A B=D .
$$

Так как это соотношение выполняется независимо от значений отдельных составляющих, то можно по определению считать, что сами наблюдаемые удовлетворяют такому соотношению:

$$
\hat{A} \widehat{B}=\widehat{D}
$$

Аналогичным образом можно определить операцию сложения двух совместимых наблюдаемых и операцию умножения наблюдаемой на действительное число.

Для фиксированной физической системы с помощью совместимых измерений каждой наблюдаемой $\hat{A} \in \mathfrak{Q}_{\xi}$ сопоставим результат измерения:

$$
\hat{A} \rightarrow A=\varphi_{\xi}(\hat{A}) .
$$

Тем самым мы зададим на алгебре $\mathfrak{Q}_{\xi}$ функционал $\varphi_{\xi}(\hat{A})$. В силу определения алгебраических операций в $\mathfrak{Q}_{\xi}$ этот функционал будет гомоморфным отображением алгебры $\mathfrak{Q}_{\xi}$ в множество действительных чисел. Такой функционал называется характером действительной коммутативной ассоциативной алгебры (см., например, [3]).

Характеры обладают следующими свойствами:

$$
\begin{aligned}
\varphi_{\xi}(\hat{I}) & =1, \\
\varphi_{\xi}(\hat{0}) & =0, \\
\varphi_{\xi}\left(\hat{A}^{2}\right) & \geqslant 0 .
\end{aligned}
$$

Здесь $\hat{I}, \hat{0}, \hat{A}$ - элементы алгебры $\mathfrak{Q}_{\xi}$ : единичный, нулевой, любой, соответственно.

При каждом реальном измерении мы всегда получаем конечный результат. Поэтому можно считать физическими только такие наблюдаемые $\hat{A}$, для которых выполняется неравенство

$$
\sup _{\xi} \sup _{\varphi_{\xi}}\left|\varphi_{\xi}(\hat{A})\right|<\infty .
$$

При выполнении этого условия характеры дополнительно обладают свойствами (см., например, [4])

$$
\varphi_{\xi}(\hat{A})=\lambda \in \sigma\left(\hat{A} ; \mathfrak{Q}_{\xi}\right),
$$

если $\lambda \in \sigma\left(\hat{A} ; \mathfrak{Q}_{\xi}\right)$, то найдется такой характер $\varphi_{\xi}(\hat{A})$, что $\lambda=\varphi_{\xi}(\hat{A})$.

Здесь $\sigma\left(\hat{A}, \mathfrak{Q}_{\xi}\right)$ - спектр элемента $\hat{A}$ в алгебре $\mathfrak{Q}_{\xi}$. Напомним, что спектром $\sigma(\hat{A}, \mathfrak{A})$ элемента $\hat{A}$ в алгебре $\mathfrak{A}$ называется множество таких чисел $\lambda$, для которых в алгебре $\mathfrak{A}$ не существует элемента, обратного элементу $\hat{A}-\lambda \hat{I}$. Свойства (4) и (5) позволяют для каждой алгебры $\mathfrak{Q}_{\xi}$ построить все ее характеры [2].

Математическим образом физической системы является множество наблюдаемых этой системы. В дальнейшем будем отождествлять физическую систему с множеством ее наблюдаемых, в котором зафиксированы соотношения между наблюдаемыми. Подмножество наблюдаемых отождествим с соответствующей физической 
подсистемой. При этом не будем предполагать, что подсистема должна быть обязательно каким-либо образом изолирована от остальной части системы. Подсистема может не быть пространственно выделена и может взаимодействовать с другими частями.

Обсудим теперь понятие состояния физической системы. Рассмотрим сначала классическую систему. В этом случае под состоянием системы понимается такой атрибут физической системы, который однозначно предопределяет результаты измерений всех наблюдаемых. Математически состояние обычно задается с помощью точки в фазовом пространстве. Однако нетрудно сообразить, что это всего лишь один из конкретных вариантов фиксирования определенного функционала на алгебре наблюдаемых, который является характером этой алгебры. Чтобы не связывать себя никаким конкретным вариантом, определим состояние классической системы как характер алгебры наблюдаемых этой системы.

На случай квантовой системы это определение обобщим следующим образом. Будем рассматривать множество $\mathfrak{A}_{+}$наблюдаемых квантовой системы как совокупность подмножеств $\mathfrak{Q}_{\xi}, \xi \in \Xi$, каждое из которых является максимальным подмножеством совместимых наблюдаемых. Каждое из этих подмножеств имеет структуру действительной коммутативной ассоциативной алгебры и может рассматриваться в качестве алгебры наблюдаемых некоторой классической подсистемы квантовой системы. Эти классические подсистемы будут открытыми, но мы по-прежнему можем описывать состояние каждой из этих подсистем с помощью характера $\varphi_{\xi}(\cdot)$ соответствующей алгебры $\mathfrak{Q}_{\xi}$.

Назовем теперь элементарным состоянием физической системы совокупность $\varphi=$ $\left[\varphi_{\xi}\right], \xi \in \Xi$, функционалов $\varphi_{\xi}(\cdot)$, каждый из которых является некоторым характером соответствующей алгебры $\mathfrak{Q}_{\xi}$.

В каждом индивидуальном измерении мы можем измерить наблюдаемые, принадлежащие какой-нибудь одной из алгебр $\mathfrak{Q}_{\xi}$. Результаты такого измерения однозначно определяются соответствующим функционалом $\varphi_{\xi}(\cdot)$, принадлежащим совокупности $\varphi$. Таким образом, результат каждого индивидуального измерения наблюдаемых физической системы определяется элементарным состоянием этой системы. Это утверждение справедливо как для классической системы (в этом случае совокупность $\varphi=\left[\varphi_{\xi}\right]$ состоит из одного функционала), так и для квантовой системы (в этом случае совокупность $\varphi=\left[\varphi_{\xi}\right]$ состоит из бесконечного числа функционалов).

Отметим, что мы не предполагаем, что справедливо равенство

$$
\varphi_{\xi}(\hat{A})=\varphi_{\xi^{\prime}}(\hat{A}), \quad \text { если } \quad \hat{A} \in \mathfrak{Q}_{\xi} \cap \mathfrak{Q}_{\xi^{\prime}} .
$$

Несмотря на кажущуюся естественность такого предположения, как указано в [2], экспериментального обоснования такого равенства нет.

Невыполнение условия (6) означает, что результат измерения может зависеть не только от исследуемой системы (от ее элементарного состояния), но и от типа прибора, с помощью которого мы осуществляем измерение. Будем говорить, что измерительные приборы принадлежат к $\xi$-типу, если для системы, находящейся в элементарном состоянии $\varphi=\left[\varphi_{\xi}\right]$ результат измерения каждой наблюдаемой $\hat{A} \in \mathfrak{Q}_{\xi}$ описывается функционалом $\varphi_{\xi}(\cdot)$. Более подробно об этом сказано в [2]. 
Заметим, что многие доказательства (см., например, [5]) невозможности существования физической реальности, определяющей результат измерения, основываются на молчаливом предположении выполнения условий типа условия (6).

Конечно, для некоторых $\varphi$ условие (6) может выполняться. Если (6) справедливо для всех $\mathfrak{Q}_{\xi}$, содержащих $\hat{A}$, то будем говорить, что элементарное состояние $\varphi=\left[\varphi_{\xi}\right]$ стабильно на наблюдаемой $\hat{A}$.

Экспериментально элементарное состояние квантовой системы однозначно зафиксировать нельзя, так как в одном эксперименте (или в группе совместимых экспериментов) самое большое, что можно измерить, - это наблюдаемые, принадлежащие одной алгебре $\mathfrak{Q}_{\xi}$. Иными словами, можно найти только значения функционала $\varphi_{\xi}(\cdot)$. В остальном элементарное состояние $\varphi$ останется неопределенным. Для определения значений других наблюдаемых придется проводить дополнительный эксперимент с использованием прибора, не совместимого с ранее использованным. Новый прибор неконтролируемым образом возмутит элементарное состояние, возникшее после первого измерения. Поэтому информация, полученная в первом эксперименте, станет бесполезной.

В связи с этим удобно объединить элементарные состояния $\varphi$, имеющие одно и то же сужение на алгебру $\mathfrak{Q}_{\xi}$ (т.е. функционал $\left.\varphi_{\xi}(\cdot)\right)$, в класс $\varphi_{\xi}$-эквивалентных элементарных состояний. Таким образом, в квантовом измерении можно установить только класс эквивалентности, к которому принадлежит элементарное состояние рассматриваемой системы.

В воспроизводимом измерении наблюдаемых, принадлежащих алгебре $\mathfrak{Q}_{\xi}$, можно узнать процедуру приготовления квантового состояния в стандартной квантовой механике. Соответственно назовем квантовым состоянием $\Psi_{\varphi_{\xi}}$ класс $\{\varphi\}_{\varphi_{\xi}} \varphi_{\xi^{-}}$ эквивалентных элементарных состояний $\varphi$, стабильных на подалгебре $\mathfrak{Q}_{\xi}$.

\section{3. ТЕОРИЯ ВЕРОЯТНОСТЕЙ В КВАНТОВОЙ МЕХАНИКЕ}

Вопреки устоявшемуся мнению о том, что колмогоровская теория вероятностей [6] не годится для квантовых систем, мы попытаемся ее использовать. По этому поводу см. [2], [7].

Базовым понятием колмогоровской теории вероятностей является вероятностное пространство [6], [8]. Это триада $(\Omega, \mathcal{F}, P)$. Первый член триады $\Omega$ - это множество (пространство) элементарных событий. Определяющими свойствами элементарных событий являются следующие: а) в каждом испытании реализуется одно и только одно элементарное событие; б) элементарные события являются взаимоисключающими. Так как два неортогональных квантовых состояния не являются взаимоисключающими, то они не могут играть роль элементарных событий. В нашем случае в качестве элементарного события будет выступать элементарное состояние $\varphi$.

Помимо элементарного события вводится еще понятие события. Каждое событие $F$ отождествляется с некоторым подмножеством множества $\Omega$. Считается, что произошло событие $F$, если реализовалось одно из элементарных событий, принадлежащих этому подмножеству $(\varphi \in F)$. Наборы подмножеств $F$ множества $\Omega$ наделяются структурой булевых $\sigma$-алгебр. 
Напомним, что булевой алгеброй множества $\Omega$ называется система подмножеств этого множества, для которой определены три алгебраические операции: объединение подмножеств, пересечение подмножеств и дополнение каждого подмножества до множества $\Omega$. Алгебра называется замкнутой относительно некоторой алгебраической операции, если в результате этой операции мы получаем элемент исходной алгебры. Булева алгебра называется $\sigma$-алгеброй, если она обладает следующими свойствами: во-первых, она содержит само множество $\Omega$ и пустое множество $\varnothing$, во-вторых, она замкнута относительно операции дополнения и счетного числа операций объединений и пересечений. Соответственно вторым членом триады является некоторая булева $\sigma$-алгебра $\mathcal{F}$.

Наконец, третий член триады - вероятностная мера $P$. Это отображение множества $\mathcal{F}$ в множество действительных чисел (каждому $F \in \mathcal{F}$ ставится в соответствие число $P(F)$ ), удовлетворяющее условиям $0 \leqslant P(F) \leqslant 1$ для всех $F \in \mathcal{F} ; \quad P(\Omega)=1$; $P\left(\sum_{j} F_{j}\right)=\sum_{j} P\left(F_{j}\right)$ для любой счетной совокупности непересекающихся подмножеств $F_{j} \in \mathcal{F}$. Вероятностная мера определяется только для событий, входящих в алгебру $\mathcal{F}$. Для элементарных событий вероятность, вообще говоря, может не существовать.

С физической точки зрения выбор определенной $\sigma$-алгебры $\mathcal{F}$ диктуется характеристиками используемой измерительной аппаратуры. Дело в том, что реальные измерительные приборы имеют конечную разрешающую способность. Поэтому они не всегда могут отличить одно элементарное событие от другого. С их помощью можно только установить, что в данном эксперименте мы имели дело с одним из элементарных событий, принадлежащим некоторому подмножеству.

В этом пункте имеется коренное различие между классическими и квантовыми физическими системами. В классическом случае мы можем неограниченно повышать разрешающую способность и использовать приборы, которые позволяют одновременно измерить значения произвольного числа наблюдаемых.

В квантовом случае совместимые измерения можно провести только для наблюдаемых, принадлежащих какой-то одной алгебре $\mathfrak{Q}_{\xi}$. Таким измерениям соответствует определенный тип $\sigma$-алгебры. Обозначим его символом $\mathcal{F}_{\xi}$. Элементы такой $\sigma$-алгебры различаются значениями (интервалами значений) наблюдаемых из алгебры $\mathfrak{Q}_{\xi}$. Более детальные измерения, в которых дополнительно измеряются значения наблюдаемых, не принадлежащих $\mathfrak{Q}_{\xi}$, являются недопустимыми, так как они не совместимы с предыдущими измерениями. Поэтому бесполезны $\sigma$-алгебры, в которых элементы дополнительно различаются по значениям наблюдаемых, не принадлежащих $\mathfrak{Q}_{\xi}$. Таким $\sigma$-алгебрам не соответствуют никакие вероятностные меры.

В связи с этим обратим внимание на тот факт, что при доказательстве (см., например, [9]) знаменитого неравенства Белла [10] молчаливо предполагалось, что вероятностная мера всегда существует. Для квантовых систем, для которых доказывается это неравенство, используемые вероятностные меры как раз не существуют [7]. Поэтому для таких физических систем вовсе не обязательно выполнение неравенства Белла. 
Выбор определенной $\sigma$-алгебры $\mathcal{F}_{\xi}$, как говорят математики, превращает множество $\Omega$ элементарных событий в измеримое пространство $\left(\Omega, \mathcal{F}_{\xi}\right)$. Этому пространству в эксперименте соответствует пара - исследуемый физический объект и определенный тип измерительной аппаратуры, допускающей совместимые измерения наблюдаемых из алгебры $\mathfrak{Q}_{\xi}$.

Действительной случайной величиной будем считать измеримое отображение измеримого пространства $\left(\Omega, \mathcal{F}_{\xi}\right)$ элементарных событий в множество действительных чисел. В приложении к наблюдаемой $\hat{A}$ это будет выглядеть так

$$
\varphi \stackrel{\hat{A}}{\longrightarrow} A_{\xi}(\varphi) \equiv \varphi_{\xi}(\hat{A}) \in \mathbb{R} \equiv[-\infty,+\infty]
$$

Обратим внимание на то, что в квантовом случае значение действительной случайной величины может зависеть не только от элементарного события (элементарного состояния), но и от типа измерительного прибора (индекса $\xi$ ).

Множество физических систем, которые описываются одним и тем же множеством $\mathfrak{A}_{+}$наблюдаемых и находятся в некотором квантовом состоянии, назовем квантовым ансамблем. Смесь квантовых ансамблей, в которую каждый из этих ансамблей входит с определенной кратностью, назовем смешанным квантовым ансамблем.

Опыт показывает, что квантовый (смешанный) ансамбль обладает вероятностными свойствами. Значит, он должен допускать введение структуры вероятностного пространства. В результате воспроизводимого измерения квантовый ансамбль переходит в новый квантовый ансамбль с другим вероятностным распределением.

Рассмотрим квантовый ансамбль систем, находящихся в квантовом состоянии $\Psi_{\varphi_{\eta}}, \eta \in \Xi$. Пространством $\Omega\left(\varphi_{\eta}\right)$ элементарных событий для такого ансамбля будет класс эквивалентности $\{\varphi\} \varphi_{\eta}$. Пусть в эксперименте используется прибор типа $\xi$. Такой ситуации соответствует измеримое пространство $\left(\Omega\left(\varphi_{\eta}\right), \mathcal{F}_{\xi}\right)$ и вероятностная мера $P_{\xi}$.

Будем измерять наблюдаемую $\hat{A} \in \mathfrak{Q}_{\xi}$ и будем считать, что в эксперименте реализуется событие $F_{A}$, если зарегистрированное значение наблюдаемой $\hat{A}$ не больше $A$. Вероятность этого события обозначим $P_{\xi}(A)=P\left(\varphi: \varphi_{\xi}(\hat{A}) \leqslant A\right)$. Если наблюдаемая $\hat{A}$ принадлежит также и алгебре $\mathfrak{Q}_{\xi^{\prime}}$, то для определения вероятности события $F_{A}$ мы могли бы использовать прибор $\xi^{\prime}$-типа. В этом случае для вероятности мы могли бы получить другое значение $P_{\xi^{\prime}}(A)$. Однако опыт показывает, что в этом случае мы получим ту же вероятность, т.е.

$$
P\left(\varphi: \varphi_{\xi}(\hat{A}) \leqslant A\right)=P\left(\varphi: \varphi_{\xi^{\prime}}(\hat{A}) \leqslant A\right) .
$$

Введем обозначение

$$
P_{\hat{A}}(d \varphi)=P(\varphi: \varphi(\hat{A}) \leqslant A+d A)-P(\varphi: \varphi(\hat{A}) \leqslant A) .
$$

Здесь мы опустили у функционала $\varphi(\hat{A})$ индекс $\xi$, имея в виду равенство $(7)$.

Для того чтобы найти среднее значение наблюдаемой $\hat{A}$ в квантовом состоянии $\Psi_{\varphi_{\eta}}$, нам нет необходимости рассматривать наблюдаемые, несовместимые с $\hat{A}$. 
Поэтому вместо рассмотрения квантовой системы мы можем ограничиться рассмотрением ее классической подсистемы, наблюдаемые которой описываются алгеброй $\mathfrak{Q}_{\xi}, \quad \hat{A} \in \mathfrak{Q}_{\xi}$. В этом случае для определения среднего значения $\langle\hat{A}\rangle$ мы можем воспользоваться математическим аппаратом классической теории вероятностей (см., например, [8]) и написать

$$
\langle\hat{A}\rangle=\int_{\varphi \in \Psi_{\varphi_{\eta}}} P_{\hat{A}}(d \varphi) A(\varphi) \equiv \int_{\varphi \in \Psi_{\varphi_{\eta}}} P_{\hat{A}}(d \varphi) \varphi(\hat{A}) \equiv \Psi_{\varphi_{\eta}}(\hat{A}) .
$$

Здесь опять-таки, имея в виду равенство (7), мы можем у функционалов $\varphi(\hat{A})$ и $A(\varphi)$ опустить индекс $\xi$.

Формула (8) определяет функционал $\Psi_{\varphi_{\eta}}(\hat{A})$ (квантовое среднее) на множестве $\mathfrak{A}_{+}$. Отметим, что

$$
\Psi_{\varphi_{\eta}}(\hat{I})=\int_{\varphi \in \Psi_{\varphi_{\eta}}} P_{\hat{I}}(d \varphi)=1 .
$$

Очевидно, что $\Psi_{\varphi_{\eta}}(\alpha \hat{A})=\alpha \Psi_{\varphi_{\eta}}(\hat{A})$, где $\alpha$ - любое действительное число. Опыт также показывает, что для любых $\hat{A} \in \mathfrak{A}_{+}$и $\widehat{B} \in \mathfrak{A}_{+}$найдется такая наблюдаемая $\widehat{D} \in \mathfrak{A}_{+}$, что для каждого квантового состояния $\Psi \varphi_{\eta}(\cdot)$ будет справедливо равенство

$$
\Psi \varphi_{\eta}(\hat{A})+\Psi \varphi_{\eta}(\widehat{B})=\Psi \varphi_{\eta}(\widehat{D}) .
$$

Такой элемент $\widehat{D}$ можно по определению считать суммой элементов $\hat{A}$ и $\widehat{B}$. Это означает, что множество $\mathfrak{A}_{+}$можно оснастить структурой действительного линейного пространства, при этом функционалы $\Psi \varphi_{\eta}(\cdot)$ линейны на этом пространстве. Благодаря свойству (3) эти функционалы положительны.

Так как любая наблюдаемая $\hat{A} \in \mathfrak{A}_{+}$совместима сама с собой, то в множестве $\mathfrak{A}_{+}$ операцию возведения $\hat{A}$ в квадрат можно определить по той же схеме, что в $\mathfrak{Q}_{\xi}$. Это позволит оснастить линейное пространство $\mathfrak{A}_{+}$структурой действительной алгебры Йордана [11], [12], в которой произведение элементов $\hat{A}$ и $\widehat{B}$ определяется формулой

$$
\hat{A} \circ \widehat{B}=\frac{(\hat{A}+\widehat{B})^{2}-\hat{A}^{2}-\widehat{B}^{2}}{2} .
$$

Это произведение очевидным образом коммутативно, но в общем случае не ассоциативно.

Все алгебры Йордана разделяются на два класса: специальные и исключительные. Алгебра Йордана называется специальной, если выполняются два условия. Во-первых, существует ассоциативная (не обязательно действительная и коммутативная) алгебра $\mathfrak{A}$ такая, что как линейное пространство, множество $\mathfrak{A}_{+}$является подпространством $\mathfrak{A}$. В алгебре $\mathfrak{A}$ помимо исходного ассоциативного произведения $\widehat{U} \widehat{V}$ можно ввести произведение с помощью формулы (10), которая в данном случае сведется к

$$
\widehat{U} \circ \widehat{V}=\frac{\widehat{U} \widehat{V}+\widehat{V} \widehat{U}}{2}
$$

Относительно такого произведения множество $\mathfrak{A}$ будет алгеброй Йордана. Во-вторых, как алгебра Йордана, $\mathfrak{A}_{+}$должна быть подалгеброй $\mathfrak{A}$. 
Можно ли использовать исключительные алгебры Йордана в квантовой физике, неизвестно. Во всех рассмотренных к настоящему времени квантовых моделях множество наблюдаемых можно оснастить структурой специальной алгебры Йордана.

Следуя этому историческом опыту, примем следующую гипотезу.

ГиПотезА. Существует инволютивная, ассоииативная и в общем случае некоммутативная алгебра $\mathfrak{A}$, удовлетворяющая следующим условиям:

а) для каждого элемента $\widehat{U} \in \mathfrak{A}$ найдется эрмитов элемент $\hat{A}$ такой, что $\widehat{U} * \widehat{U}=\hat{A}^{2}$

б) если $\widehat{U}^{*} \widehat{U}=0$, mo $\widehat{U}=0$;

в) множество эрмитовых элементов алгебры $\mathfrak{A}$ совпадает с множеством $\mathfrak{A}_{+}$ наблюдаемых.

Принимая эту гипотезу, мы вынуждены отступить от строгого соблюдения правила делать только те предположения, необходимость которых продиктована физическим опытом. Нам придется довольствоваться менее надежным критерием историческим опытом. Вместе с тем надо подчеркнуть, что в стандартной квантовой механике помимо этой гипотезы делается гораздо менее очевидное предположение: предполагается, что наблюдаемые являются самосопряженными операторами в некотором гильбертовом пространстве.

Далее физическую систему будем считать заданной, если задана алгебра $\mathfrak{A}$. Так как алгебры $\mathfrak{Q}_{\xi}$ совместимых наблюдаемых являются максимальными действительными коммутативными подалгебрами алгебры $\mathfrak{A}$, принадлежащими $\mathfrak{A}_{+}$, то совместимые наблюдаемые являются взаимно коммутирующими элементами алгебры $\mathfrak{A}$, а несовместимые наблюдаемые не коммутируют между собой.

\section{4. ДИНАМИЧЕСКИЕ ВЕЛИЧИНЫ И $C^{*}$-АЛГЕБРА}

Элементы алгебры $\mathfrak{A}$ в дальнейшем будем называть динамическими величинами. Любой элемент $\widehat{U} \in \mathfrak{A}$ однозначно представляется в виде $\widehat{U}=\hat{A}+i \widehat{B}$, где $\hat{A}, \widehat{B} \in \mathfrak{A}_{+}$. Поэтому функционал $\Psi_{\varphi_{\eta}}(\cdot)$ можно однозначно расширить до линейного функционала на алгебре $\mathfrak{A}: \Psi_{\varphi_{\eta}}(\widehat{U})=\Psi_{\varphi_{\eta}}(\hat{A})+i \Psi_{\varphi_{\eta}}(\widehat{B})$.

Как показано в [2], справедливо равенство

$$
\sup _{\eta} \sup _{\varphi_{\eta}} \Psi_{\varphi_{\eta}}\left(\widehat{U}^{*} \widehat{U}\right)=\sup _{\eta} \sup _{\varphi_{\eta}} \varphi_{\eta}\left(\widehat{U}^{*} \widehat{U}\right)
$$

Поэтому в алгебре $\mathfrak{A}$ можно ввести норму с помощью равенства [2], [12]

$$
\|\widehat{U}\|^{2}=\sup _{\eta} \sup _{\varphi_{\eta}} \varphi_{\eta}\left(\widehat{U}^{*} \widehat{U}\right)
$$

Так как функционал $\varphi_{\eta}$ является характером алгебры $\mathfrak{Q}_{\eta}$, то мы имеем равенство $\varphi_{\eta}\left(\left[\widehat{U}^{*} \widehat{U}\right]^{2}\right)=\left[\varphi_{\eta}\left(\widehat{U}^{*} \widehat{U}\right)\right]^{2}$. Отсюда следует, что

$$
\|\widehat{U} * \widehat{U}\|=\|\widehat{U}\|^{2}
$$


Полная нормированная инволютивная алгебра, в которой норма удовлетворяет дополнительному условию (11), называется $C^{*}$-алгеброй [3]. Таким образом, алгебра квантовых динамических величин может быть оснащена структурой $C^{*}$-алгебры.

Замечательной особенностью $C^{*}$-алгебры является то, что любая $C^{*}$-алгебра изометрически изоморфна подалгебре линейных ограниченных операторов в подходящем гильбертовом пространстве $\mathfrak{H}[3]$. Говорят, что в пространстве $\mathfrak{H}$ реализуется точное представление $C^{*}$-алгебры. Это позволяет включить в предлагаемую в настоящей статье схему математический аппарат стандартной квантовой механики.

Связь $C^{*}$-алгебры с гильбертовым пространством реализует так называемая каноническая конструкция Гельфанда-Наймарка-Сигала (ГНС-конструкция), см., например, [12], [13]. Она состоит в следующем.

Пусть имеются некоторая $C^{*}$-алгебра $\mathfrak{A}$ и линейный положительный функционал $\Psi$ на этой алгебре. Будем считать два элемента $\widehat{U}, \widehat{U}^{\prime} \in \mathfrak{A}$ эквивалентными, если для любого $\widehat{W} \in \mathfrak{A}$ справедливо равенство $\Psi\left(\widehat{W}^{*}\left(\widehat{U}-\widehat{U}^{\prime}\right)\right)=0$. Обозначим через $\Phi(\widehat{U})$ класс эквивалентности элемента $\widehat{U}$ и рассмотрим множество $\mathfrak{A}(\Psi)$ всех классов эквивалентности в $\mathfrak{A}$. Превратим множество $\mathfrak{A}(\Psi)$ в линейное пространство, положив $a \Phi(\widehat{U})+b \Phi(\widehat{V})=\Phi(a \widehat{U}+b \widehat{V})$. Определим в $\mathfrak{A}(\Psi)$ скалярное произведение формулой

$$
(\Phi(\widehat{U}), \Phi(\widehat{V}))=\Psi\left(\widehat{U}^{*} \widehat{V}\right) .
$$

Это скалярное произведение порождает в алгебре $\mathfrak{A}(\Psi)$ норму $\|\Phi(\widehat{U})\|=\left[\Psi\left(\widehat{U}^{*} \widehat{U}\right)\right]^{1 / 2}$. Пополнение по этой норме превращает $\mathfrak{A}(\Psi)$ в гильбертово пространство. Каждый элемент $\widehat{V}$ алгебры $\mathfrak{A}$ однозначно представляется в этом пространстве линейным оператором П $(\widehat{V})$, действующим по правилу

$$
\Pi(\widehat{V}) \Phi(\widehat{U})=\Phi(\widehat{V} \widehat{U})
$$

Таким образом, ГНС-конструкция порождает представление $C^{*}$-алгебры линейными операторами в гильбертовом пространстве.

Рассмотрим ГНС-конструкцию, в которой в качестве функционала, порождающего представление, фигурирует $\Psi_{\varphi_{\eta}}(\widehat{B})$. Пусть $\Phi(\hat{I})$ - класс эквивалентности элемента $\hat{I}$, тогда согласно равенствам (12) и (13) получаем, что

$$
(\Phi(\hat{I}), \Pi(\widehat{B}) \Phi(\hat{I}))=\Psi_{\varphi_{\eta}}(\widehat{B})
$$

для любого $\widehat{B} \in \mathfrak{A}$.

Согласно равенству $(8)$ функционал $\Psi_{\varphi_{\eta}}(\widehat{B})$ описывает среднее значение наблюдаемой $\widehat{B}$ в квантовом состоянии $\Psi_{\varphi_{\eta}}$. Равенство (14) говорит о том, что это среднее значение равняется математическому ожиданию оператора $\Pi(\widehat{B})$ в состоянии, описываемом вектором $\Phi(\hat{I})$ гильбертова пространства. Это позволяет в предлагаемом подходе для вычисления квантовых средних в полной мере использовать математический аппарат стандартной квантовой механики.

Вместе с тем здесь имеется существенное отличие предлагаемого подхода от стандартной квантовой механики. В последней соотношение типа (14) постулируется 
(постулат Борна [14]) и является отправным пунктом для построения так называемой квантовой теории вероятностей. Впрочем, в отличие от классической, квантовая теория вероятностей как стройная математическая схема до сих пор не построена. В данной статье формула (14) получена как следствие физически обоснованных утверждений и классической теории вероятностей. Кроме того, указано, когда она справедлива. А именно формула (14) применима для вычисления средних значений наблюдаемых по квантовому ансамблю.

\section{5. “ДА-НЕТ” ЭКСПЕРИМЕНТ, КОЛЛАПС КВАНТОВОГО СОСТОЯНИЯ}

Пусть $\mathbb{R}=[-\infty,+\infty]$ - расширенная действительная прямая, а $\mathcal{S} \subset \mathbb{R}-$ некоторый интервал или объединение счетного числа интервалов.

Пусть в эксперименте измеряется некоторая наблюдаемая физической системы. Будем говорить, что в эксперименте получен ответ "да", если измеренное значение наблюдаемой оказалось внутри $\mathcal{S}$, и получен ответ "нет", если значение оказалось вне $\mathcal{S}$. Такая процедура называется "да-нет" экспериментом. Фактически любой реальный эксперимент сводится либо к “да-нет” эксперименту, либо к последовательности таких экспериментов.

Можно считать, что в “да-нет" эксперименте измеряется значение специальной наблюдаемой величины. В качестве этой наблюдаемой примем такое свойство исследуемой системы, которое вызывает либо ответ “да" (в этом случае этой наблюдаемой приписывается значение 1), либо ответ "нет" (в этом случае наблюдаемой приписывается значение 0). Если измерение воспроизводимо, то такая наблюдаемая будет обладать свойствами проектора $\hat{p}$. Напомним, что проектором называется любой элемент алгебры, который удовлетворяет условиям: $\hat{p}^{*}=\hat{p}, \hat{p}^{2}=\hat{p}$.

Одним из основных постулатов стандартной квантовой механики является так называемый проекционный принцип [1]. В простейшей форме он выглядит следующим образом. Пусть физическая система находится в квантовом состоянии, которое описывается нормированным вектором $|\Psi\rangle$ некоторого гильбертова пространства $\mathfrak{H}$. Пусть производен "да-нет" эксперимент (измеряется наблюдаемая $\hat{p}$ ) и получен ответ "да". Тогда в результате эксперимента физическая система переходит в новое квантовое состояние, которое описывается вектором $\left|\Psi^{\prime}\right\rangle=\hat{p}|\Psi\rangle[\langle\Psi|\hat{p}| \Psi\rangle]^{-1 / 2}$.

Эквивалентно это утверждение можно выразить так. Векторы $|\Psi\rangle$ и $\left|\Psi^{\prime}\right\rangle$ описывают квантовые состояния, которым соответствуют линейные функционалы $\Psi(\hat{A})=$ $\langle\Psi|\hat{A}| \Psi\rangle$ и $\Psi^{\prime}(\hat{A})=\left\langle\Psi^{\prime}|\hat{A}| \Psi^{\prime}\right\rangle$, где $\hat{A}$ - любая наблюдаемая. Отождествим квантовое состояние с соответствующим функционалом. Тогда в результате описанного эксперимента физическая система из квантового состояния $\Psi(\hat{A})$ перейдет в квантовое состояние $\Psi^{\prime}(\hat{A})$ :

$$
\Psi(\hat{A}) \rightarrow \Psi^{\prime}(\hat{A})=\Psi(\hat{p} \hat{A} \hat{p})[\Psi(\hat{p})]^{-1}
$$

Общая форма проекционного принципа сводится к постулированию равенства (15) для произвольных квантовых состояний, т.е. для состояний, которые описываются 
любыми линейными положительными нормированными функционалами. Физическое явление, которое управляется проекционным принципом, получило название коллапса (редукции) квантового состояния.

Проекционный принцип широко и успешно используется для описания воздействия измерительных приборов на квантовую систему. Вместе с тем коллапс квантового состояния как физическое явление резко противоречит нашим интуитивным представлениям.

Например, физическая система, находящаяся в состоянии (почти) плоской волны, размазана (почти) по всему координатному пространству. В результате измерения координаты эта система (почти) мгновенно стягивается в (почти) точку. Существуют многочисленные рецепты преодоления этого парадокса. Но все они выглядят, мягко выражаясь, не очень убедительно.

Предлагаемый в настоящей работе подход позволяет отказаться от проекционного принципа как самостоятельного постулата. Вместо этого оказывается возможным получить соотношение (15) как следствие приложения классической теории вероятностей к сформулированным ранее утверждениям, которые не противоречат физической интуиции.

Итак, пусть физическая система находится в квантовом состоянии $\Psi$, которое описывается линейным функционалом $\Psi(\hat{A})$. Пусть прибор осуществляет воспроизводимое измерение наблюдаемой $\hat{p}$, которая является проектором, и результат измерения равен 1 .

Рассматриваемая физическая система является элементом соответствующего квантового ансамбля. Мысленно представим, что аналогичную процедуру мы повторили с другими элементами этого ансамбля. В результате таких измерений квантовый ансамбль, соответствующий квантовому состоянию $\Psi$, перейдет в другой квантовый ансамбль. Этот новый ансамбль соответствует квантовому состоянию $\Psi^{\prime}$, а средние значения наблюдаемых $\hat{A}$ по этому ансамблю будет определяться функционалом $\Psi^{\prime}(\hat{A})$.

Этот функционал получается процедурой усреднения (см. формулу (8)) функционалов $\varphi$, соответствующих элементарным состояниям. Каждое элементарное состояние $\varphi$, входящее в квантовое состояние $\Psi^{\prime}$, будет устойчиво на наблюдаемой $\hat{p}$, причем $\varphi(\hat{p})=1$. Благодаря этому и равенству (9) функционал $\Psi^{\prime}$ будет удовлетворять условию

$$
\Psi^{\prime}(\hat{p})=\Psi^{\prime}(\hat{I})=1 .
$$

Так как $\Psi^{\prime}(\hat{A})$ является положительным линейным функционалом, то для него справедливо неравенство Коши-Буняковского-Шварца. Поэтому

$$
\left|\Psi^{\prime}(\hat{A}(\hat{I}-\hat{p}))\right|^{2} \leqslant \Psi^{\prime}\left(\hat{A}^{*} \hat{A}\right) \Psi^{\prime}(\hat{I}-\hat{p}) .
$$

В силу (16) правая часть этого неравенства равна нулю. Отсюда

$$
\Psi^{\prime}(\hat{A})=\Psi^{\prime}(\hat{A} \hat{p}) .
$$

Аналогично

$$
\Psi^{\prime}(\hat{A})=\Psi^{\prime}(\hat{p} \hat{A}) .
$$


Делая в (18) замену $\hat{A} \rightarrow(\hat{I}-\hat{p}) \hat{A}$ и учитывая $(18)$ и $(19)$, получаем

$$
\Psi^{\prime}(\hat{A})=\Psi^{\prime}(\hat{p} \hat{A} \hat{p})=\frac{\Psi^{\prime}(\hat{p} \hat{A} \hat{p})}{\Psi^{\prime}(\hat{p})} .
$$

Из формулы (20) следует, что вместо того чтобы в состоянии $\Psi^{\prime}$ находить среднее значение наблюдаемой $\hat{A}$, мы можем находить среднее значение наблюдаемой $\hat{A}_{p} \equiv$ $\hat{p} \hat{A} \hat{p}$.

Так как $\left[\hat{A}_{p}, \hat{p}\right]=0$, то наблюдаемые $\hat{A}_{p}$ и $\hat{p}$ совместимы. В силу формулы (7) вероятностные распределения, а значит, и средние значения наблюдаемых, не зависят от типа измерительного прибора, который используется для определения значений этих величин. Поэтому в дальнейших рассуждениях мы можем ограничиться рассмотрением совместимых измерений наблюдаемых $\hat{A}_{p}$ и $\hat{p}$. Такие измерения должны проводиться приборами, принадлежащими какому-то одному типу $\eta$, которому соответствует максимальная действительная коммутативная подалгебра $\mathfrak{Q}_{\eta}$ алгебры наблюдаемых, содержащая наблюдаемые $\hat{A}_{p}$ и $\hat{p}$.

Будем интересоваться средним значением наблюдаемой $\hat{A}_{p}$ при условии, что значение наблюдаемой $\hat{p}$ равно 1. Поскольку другие наблюдаемые нас не интересуют, то вместо рассмотрения полной квантовой системы мы можем ограничиться рассмотрением ее классической подсистемы, все наблюдаемые которой принадлежат подалгебре $\mathfrak{Q}_{\eta}$. В этом случае мы можем воспользоваться правилами вычисления условного среднего в классической теории вероятностей.

Напомним, что условная вероятность $P(F(\mathcal{S}) \mid F(p))$ события $F(\mathcal{S})$ при условии, что реализуется событие $F(p)$, задается формулой (см., например, [11])

$$
P(F(\mathcal{S}) \mid F(p))=\frac{P(F(\mathcal{S}) \cap F(p))}{P(F(p))} .
$$

Здесь $P(F(p))$ - вероятность события $F(p)$ (предполагается, что $P(F(p)) \neq 0$ ), а $P(F(\mathcal{S}) \cap F(p))$ - вероятность одновременной реализации событий $F(\mathcal{S})$ и $F(p)$.

В рассматриваемом нами случае в качестве пространства элементарных событий выступает множество элементарных состояний, принадлежащих квантовому состоянию $\Psi$. Событию $F(p)$ соответствует множество тех элементарных состояний (элементарных событий), для которых $\varphi_{\eta}(\hat{p})=1$. Событию $F(\mathcal{S})$ соответствует множество элементарных состояний, для которых $\varphi_{\eta}\left(\hat{A}_{p}\right) \in \mathcal{S}$, где $\mathcal{S}$ - описанное в начале этого раздела подмножество действительной оси; $F(\mathcal{S}) \cap F(p)$ - пересечение множеств $F(\mathcal{S})$ и $F(p)$.

Так как $\varphi_{\eta}\left(\hat{A}_{p}\right)=\varphi_{\eta}\left(\hat{p} \hat{A}_{p}\right)=\varphi_{\eta}(\hat{p}) \varphi_{\eta}\left(\hat{A}_{p}\right)$, то $\varphi_{\eta}\left(\hat{A}_{p}\right)=0$, если $\varphi_{\eta}(\hat{p})=0$. Поэтому среднее значение наблюдаемой $\hat{A}_{p}$, вычисленное с помощью вероятностной меры $P(F(\mathcal{S}) \cap F(p))$, совпадает со средним значением, вычисленным с помощью меры $P(F(\mathcal{S}))$. С другой стороны, поскольку $\varphi_{\eta}(\hat{p})$ равно либо 1 , либо 0 , то $P(F(p))$ равно среднему значению наблюдаемой $\hat{p}$.

Поэтому из равенства (21) следует, что условное среднее наблюдаемой $\hat{A}_{p}$ определяется формулой

$$
\left\langle\hat{A}_{p} \mid F(p)\right\rangle=\frac{\Psi\left(\hat{A}_{p}\right)}{\Psi(\hat{p})}=\frac{\Psi(\hat{p} \hat{A} \hat{p})}{\Psi(\hat{p})} .
$$


По своему физическому смыслу квантовое среднее $\Psi^{\prime}(\hat{p} \hat{A} \hat{p})$, которое фигурирует в формуле (20), совпадает с условным средним $\left\langle\hat{A}_{p} \mid F(p)\right\rangle$. Поэтому формулу (20) можно переписать в виде

$$
\Psi^{\prime}(\hat{A})=\frac{\Psi(\hat{p} \hat{A} \hat{p})}{\Psi(\hat{p})},
$$

т.е. мы приходим к формуле (15), которая в стандартной квантовой механике является математической формулировкой проекционного принципа. В отличие от формулы (15) формула (22) является следствием только интуитивно понятных утверждений и классической теории вероятностей. Отметим также, что формула (22) относится к квантовому ансамблю (квантовому состоянию), а не к отдельному члену этого ансамбля (элементарному состоянию). Поэтому, когда мы говорим, например, что некоторая квантовая частица находится в состоянии плоской волны, то это означает, что ее элементарное состояние принадлежит множеству элементарных состояний, для которых значение импульса одно и то же, а значения координат разных членов ансамбля разные. Это не означает, что рассматриваемая частица размазана по всему координатному пространству. Элементарное состояние конкретной частицы может быть таким, что значения координаты локализованы в достаточно малой области. Этих значений может быть много, так как это элементарное состояние не обязано быть стабильным по координате. При измерении координаты этой частицы мы не стягиваем плоскую волну в точку. Мы просто определяем область локализации координаты у ее элементарного состояния. Конечно, в результате воздействия измерительного прибора элементарное состояние частицы изменится. Во-первых, если измерение воспроизводимо, то состояние станет стабильным по координате. Во-вторых, изменится значение импульса. Однако это не означает, что частица станет размазанной в импульсном пространстве. Просто ее элементарное состояние станет членом другого квантового ансамбля, который содержит элементарные состояния, соответствующие различным значениям импульса.

\section{6. ЗАКЛЮЧЕНИЕ}

Описанный в настоящей статье подход к квантовой теории ни в коей мере не отвергает стандартную квантовую механику. Отцы-основатели квантовой механики построили замечательное здание. Но они начали возводить это здание со второго этажа - с описания вероятностей и средних значений. Поэтому для устойчивости этого здания потребовалось большое количество подпорок в виде целого ряда "принципов": принцип суперпозиции, принцип неопределенностей, принцип дополнительности, проекционный принцип, принцип неразличимости, принцип отсутствия траекторий. Все эти принципы выглядят в достаточной мере искусственными и трудно поддаются физической интерпретации. Основная задача этих принципов оправдать математический аппарат стандартной квантовой механики. Надо сказать, что этот математический аппарат оказался на редкость удачным, чего нельзя сказать о его физической интерпретации. Недаром до сих пор не стихают споры о физической интерпретации квантовой механики, хотя сам термин “физическая интерпретация" 
представляется весьма странным. Если квантовая механика - это физическая теория, то она не должна нуждаться ни в какой физической интерпретации. Используя указанный термин, мы вольно или невольно признаем, что квантовая механика это не физическая теория, а математическая модель. В настоящей работе предпринята попытка построить квантовую механику именно как физическую теорию, основывающуюся на опытных данных.

Центральным пунктом описываемого подхода является введение понятия “элементарное состояние", которое отсутствует в аппарате стандартной квантовой механики. Это понятие, с одной стороны, дает четкий математический образ такого физического явления, как индивидуальный эксперимент. С другой стороны, оно позволяет использовать хорошо разработанный аппарат классической логики и классической теории вероятностей. При этом надо иметь в виду, что, хотя часто можно услышать ссылки на так называемую квантовую логику и квантовую теорию вероятностей, до сих пор не удалось придать им структуру четкой завершенной теоретической схемы.

Основываясь на понятии элементарного состояния и используя классическую теорию вероятностей, мы можем полностью воспроизвести математический аппарат стандартной квантовой механики, одновременно указав область его применимости. Этот аппарат применим для квантовых ансамблей. Это очень важный вид ансамблей, но далеко не самый общий. В частности, этот аппарат не годится для описания индивидуального события.

\section{Список литературы}

[1] И. Фон Нейман, Математические основы квантовой механики, Наука, М., 1964.

[2] Д. А. Славнов, ТМФ, 142 (2005), 510.

[3] Ж. Диксмье, $C^{*}$-алгебры и их представления, Наука, М., 1974.

[4] У. Рудин, Функиионалъный анализ, Мир, М., 1975.

[5] S. Kochen, E. P. Specker, J. Math. Mech., 17 (1967), 59; D. M. Greenberg, M. A. Horne, A. Shimony, A. Zeilinberg, Amer. J. Phys., 58 (1990), 1131.

[6] А.Н. Колмогоров, Основные понятия теории вероятностей, Наука, М., 1974.

[7] Д. А. Славнов, ТМФ, 136 (2003), 436.

[8] Ж. Неве, Математические основы теории вероятностей, Мир, М., 1969.

[9] J. F. Clauser, M. A. Horn, A. Shimony, R. A. Holt, Phys. Rev. Lett., 23 (1969), 880.

[10] J. S. Bell, Physics, 1 (1965), 195.

[11] P. Jordan, Z. Phys., 80 (1933), 285.

[12] Ж. Эмх, Алгебрачческий подход в статистической механике и квантовой теории поля, Мир, М., 1976.

[13] М. А. Наймарк, Нормированные кольща, Наука, М., 1968.

[14] M. Born, Z. Phys., 37 (1926), 863; 38, 803; 40 (1927), 167. 